\title{
The Seeming Interdependence Between the Concepts of Valid Inference and Proof
}

\author{
Dag Prawitz ${ }^{1,2}$
}

Published online: 4 October 2017

(C) The Author(s) 2017. This article is an open access publication

\begin{abstract}
We may try to explain proofs as chains of valid inference, but the concept of validity needed in such an explanation cannot be the traditional one. For an inference to be legitimate in a proof it must have sufficient epistemic power, so that the proof really justifies its final conclusion. However, the epistemic concepts used to account for this power are in their turn usually explained in terms of the concept of proof. To get out of this circle we may consider an idea within intuitionism about what it is to justify the assertion of a proposition. It depends on Heyting's view of the meaning of a proposition, but does not presuppose the concept of inference or of proof as chains of inferences. I discuss this idea and what is required in order to use it for an adequate notion of valid inference.
\end{abstract}

Keywords Valid inference · Proof - Epistemic ground · Intuitionism

\section{Introduction}

Proofs as we usually know them from mathematical practice are built up of inferences. So it seems natural to expect that the concept of proof can be explained somehow in terms of the concept of inference. However, we make quite different presuppositions about proofs and inferences. Proofs are supposed to establish theorems. It is not only that the concept of proof is factive or veridical in the sense that the proposition

Dag Prawitz

dag.prawitz@philosophy.su.se

1 Department of Philosophy, Stockholm University, 10691 Stockholm, Sweden

2 Engelbrektsgatan 4, 11432 Stockholm, Sweden
$A$ that occurs in the final conclusion of a proof is true, the truth of $A$ is guaranteed by the proof, that is to say, the proof supplies a ground for asserting $A$ and thereby justifies its final conclusion, the assertion of $A$.

It is quite different with the concept of inference. There are valid and invalid inferences. An attempted proof that contains an invalid inference fails in justifying its final conclusion and is then not a proof. Consequently, the concept of proof cannot be explained by saying that a proof is a chain of inferences-we have to say that it is a chain of valid inferences.

We could of course change usage, which is not very fixed anyway, and take validity to be included among the properties defining the concept of inference. But that would not change anything essential. Questions about the validity of inferences would only have to be considered at an earlier stage when explaining what it is to be an inference. I find it convenient to stick to common usage and not count validity among the defining properties of the concept of inference; it allows us among other things to speak of the inferences occurring in a line of thought without committing us to their validity.

But what is a valid inference? The traditional definition of valid inference in terms of necessary preservation of truth from premisses to conclusion is obviously inadequate here, and so is clearly the common definition of validity within model theory, first proposed by Bolzano and then by Tarski, in which the necessity is replaced by the requirement that truth is preserved under all interpretations of the non-logical terms occurring in the premisses and the conclusion. If that was all that was required of inferences of a proof, every logical consequence could be established by a proof with just one inference step, regardless of how difficult it was to see that logical consequence actually pertains; as we all know, 
to establish a consequence relation may require a proof with many steps.

It is a riddle how this inadequate way of defining the validity of inference can have come to be so widely accepted, commonly repeated in most textbooks of logic. It is sometimes said that it goes back to Aristotle's notion of syllogism. But Aristotle distinguished in his Analytica Priora between perfect and imperfect syllogisms, saying: "A perfect syllogism is one that needs nothing other than the premisses to make the conclusion evident". 1 This important notion is absent in the main stream of contemporary logic.

A definition of proof in terms of valid inference requires that the latter concept be explained in terms of evidence or related notions such as ground, justification, or knowledge; since a proof is understood in terms of such epistemic concepts, a valid inference must also be related to these concepts if it is to serve in an explanation of what a proof is.

But we cannot simply use an epistemic concept in the scheme of the traditional definition of valid inference and just require that the chosen epistemic property be preserved from premisses to conclusion. It is not enough that the property can be shown to apply to the conclusion, for instance, that the conclusion is justifiable if the premisses are-for an inference to be legitimate in a proof, the epistemic property must be mediated directly by the inference as it stands so that there is no need of another proof to see that the property is preserved.

Something more must consequently be required of an inference that is used in a proof. Per Martin-Löf (1985) speaks of the correctness of inferences and says that to convince yourself that an immediate inference is correct, "you must make the conclusion evident to yourself immediately, without any intervening steps, on the assumption that you know the premiss[es]". 2 Similarly, criticizing the traditional notion of valid inference, Göran Sundholm says: "an immediate inference is valid when the conclusion can immediately be made upon the assumption that the premisses are known"; the assumption in question he calls epistemic. ${ }^{3}$

What does then such an epistemic assumption amount to? Both Martin-Löf and Sundholm take premisses and conclusions of inferences to be assertions or judgements. Here I am interested in these speech acts when they have propositions as contents. To know a premiss is then according to these authors to know that the proposition in question is true, which Martin-Löf equates in the quoted paper with possessing a proof of the truth of the proposition.

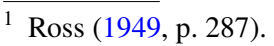

2 Martin-Löf (1996, p. 45), a reprint of Martin-Löf (1985). The italics are mine.

3 Sundholm (1997, p. 210).
}

However, if we first explain the concept of proof saying that a proof is a chain of valid inferences, and then explain the validity of an inference by referring among other things to proofs of the premisses, we are moving in a plain circle. ${ }^{4}$

It may therefore seem that not only does the concept of proof depend on the concept of valid inference, but also conversely the latter concept depends on the former. If one of the two concepts could be explained without reference to the other, the other concept would easily be defined in terms of the first. Assuming that the concept of proof is given independently of the concept of valid inference, we can define the latter as follows: An inference of $A$ from the premisses $A_{1}, A_{2}, \ldots$, and $A_{n}$ is valid, if and only if, the result of conjoining any proofs of the premisses

\section{$\mathrm{P}_{\mathrm{i}}$}

$\mathrm{A}_{\mathrm{i}}$

$(i \leq n)$ by adding the inference in question, attaching it to the end of the proofs so to say, indicated by the figure

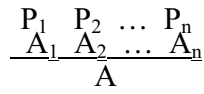

is again a proof.

Accordingly, there may be in principle two ways of breaking up the apparent interdependency between the concepts of proof and valid inference, thereby making explanatory progress with respect to the two concepts: either explaining the validity of inferences without referring to proofs or explaining the concept of proof without referring to the validity of inferences. The second alternative is in my view to put the natural conceptual order upside down. So the first alternative seems to me preferable, but is any of the two alternatives at all possible?

\section{The Problem of Defining the Validity of Inferences}

The problem that we are confronted with when trying to explain what it is for an inference to be valid is not only to account for epistemic assumptions. The more general problem is to account for the epistemic property-evidence, being warranted, justification, ground, knowledge, or how you name it - that must be transmitted by a valid inference.

\footnotetext{
${ }^{4}$ Martin-Löf drew attention to this circularity in a lecture given 2015 , saying that it had plagued him for many years. In the paper quoted above, he avoids the circularity by taking proof and inference to be the same. But later he has wanted to explain proofs as chains of valid inferences and then the circularity has arisen again.
} 
Let us first note that it is only by doing something that we can become justified in making an assertion. Therefore, being interested here in the capacity of a proof to establish theorems or justify assertions, we must primarily look at a proof as an act. The inferences that make up a proof have consequently to be seen as acts, too. We may restrict ourselves to generic acts, abstracting from the agents of the individual acts and the situations in which they are performed.

In logic we often make a further abstraction and consider an inference as individuated simply by its premisses and conclusion. To keep inferences in that sense distinct from inference acts we may call them inference figures, exhibited for instance in the form

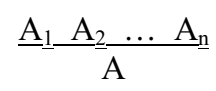

where $A_{1}, A_{2}, \ldots, A_{n}$ and $A$ are propositions or sentences. The figure can be seen as a representation of a generic inference act where we pass from premisses that assert $A_{1}, A_{2}, \ldots, A_{n}$ to a conclusion that asserts $A$.

Similarly we may speak of proof figures made up of a number of valid inference figures arranged for instance in the form of tree. Gentzen's natural deductions are such proof figures, which can be seen as representing proofs.

To get clear about what is required of a valid inference, we should look at what is going in an inference act and what its aim is. An inference involves a transition from a number of assertions, the premisses, to another assertion, the conclusion. Such transitions often occur automatically, but inferences in proofs are what we can call reflective, that is, they are consciously made with the aim to justify the conclusion, granted that the premisses have already been justified. An inference is acceptable in a proof only if this aim is attained-if it is not attained the inference constitutes a gap in an attempted proof. From outside a reflective inference appears as a complicated speech act containing a number of assertions and an indication, for instance, by the insertion of words such as "hence" or "because", that one of the assertions is seen as supported by the other ones. But the significance of an inference if successful is that the very inference act justifies the conclusion given that the premisses are already justified.

I have suggested that this is taken as a definition of what it is for an inference to be legitimate in the course of a proof (using the term "legitimate" instead of "valid", since the latter term is already in common use for another notion):

"An inference is legitimate if and only if by making the inference a subject who has already a justification of its premisses thereby gets a justification of its conclusion" (Prawitz 2011, p. 388).

This is just another formulation of the idea behind Aristotle's notion of perfect syllogism or Martin-Löf's and Sundholm's explanations quoted above of what it is for an inference to be correct or valid, avoiding the psychological ring that may be associated with Martin-Löf's formulation and making the epistemic concept that is hidden in Sundholm's formulation explicit. The common idea is that a successful inference should make the conclusion evident or warranted under an epistemic assumption about the premisses. The crucial problem remains however: can we account without circularity for the epistemic concept that must be involved in the requirement on the inferences of a proof?

Let us concentrate on the notion of ground, one of the more or less synonymous notions used to describe epistemically adequate assertions. We expect rational speakers to have sufficient grounds for their assertions, and there are various ways in which this may be fulfilled so that the assertions become warranted or justified. For instance, in the case of so-called observational statements, a relevant observation counts as a ground for the assertion; seeing that it rains is a ground for asserting that it is raining. In the case of an arithmetical identity $t=u$, to have carried out calculations of $t$ and $u$ and have found that they agree would commonly be considered to constitute a ground for asserting the identity.

A ground for an assertion may only make it more or less likely that the asserted proposition is true, but since we are here concerned with deductive proofs and deductive inferences, the grounds that we are considering should be conclusive. The problem is what counts as a conclusive ground for the assertion of a logically compound proposition. It may seem that here we cannot avoid to speak of proofs-what else could count as a conclusive ground for the assertion of, for instance, a universal proposition? In fact, the identification of grounds for theorems with deductive proofs has been the established tradition in mathematics, although none of these two concepts have been the object of much analysis within logic or philosophy of mathematics.

In the first half of the twentieth century there were however two profound ideas about proofs and justification, one due to Gentzen and one due to Heyting. They were formulated as short remarks and were never much developed by their authors. I am thinking of the remarks by Gentzen that he made after having set up his system of natural deduction and of the remarks by Heyting about the concept of proof and the concept of construction.

Gentzen's remark that his introduction rules "define" the meanings of the logical constants seems to acknowledge the point made above that grounds for compound assertions are explained in terms of what we count as proofs of them: introduction inferences are meaning constitutive, which is to say, it seems, that proofs by them are to be accepted as grounds since the meanings of their conclusions are given by saying that this is just how their conclusions are directly justified. However, this is much more subtle than identifying grounds with proofs - not any proof counts by definition as a ground for its conclusion. The proof has to end with an introduction 
inference, whose premisses and discharged assumptions, if any, have lower complexity than the conclusion, which seems to open for a recursive explanation of meaning and the concept of ground. Furthermore, other inferences are not justified in this way but by being in accord with the meaning explanations given in terms of introduction inferences.

Gentzen only exemplifies how his elimination inferences are justified in this way. The idea has to be generalized to apply not just to his elimination inferences but to inferences in general, if we want to use it to explain what it is to be a proof or a valid inference. This is attempted in what has been called proof theoretic semantics with its notion of valid argument, which however gives rise to a notion of proof not based on a notion of valid inference. It exemplifies the approach that I described above as the natural conceptual order turned upside down. Having recently discussed this notion of valid argument elsewhere,${ }^{5}$ I shall not take it up here.

Heyting proposed a notion of proof that was not at all concerned with inferences. It has given rise within intuitionism to a long discussion of various notions of proofs, which I shall review briefly below. I shall argue that neither Heyting nor his intuitionistic successors explain the concept of proof that I am discussing here but that his notion of construction may be developed so that it gives us what we are looking for, namely a concept of justification or ground not based on the concept of proof.

\section{Notions of Proofs Within Intuitionism}

Heyting's notion of proof depends on his notion of proposition, which he explained as expressing an intention of a construction that satisfies certain conditions. Like Frege, Heyting distinguished carefully between propositions and assertions. The assertion of a proposition he understood as signifying the realization of the intention expressed by the proposition, ${ }^{6}$ in other words, as saying that the intended construction has been found. In terms of these concepts he explained a proof of a proposition as consisting in "the realization of the construction required by the proposition"?

Here we have a notion of proof explained without reference to inferences. ${ }^{8}$ Nevertheless, the notion retains its usual

\footnotetext{
5 See e.g. Prawitz (2015a, 2018); this notion of valid argument was first proposed by Prawitz (1973) and was later modified by Dummett (1991) and Schroeder-Heister (2006).

6 "Die Behauptung einer Aussage bedeutet die Erfüllung der Intention." (Heyting 1931, p. 113).

7 "Ein Beweis für eine Aussage besteht in der Verwirklichung der in ihr geforderten Konstruktion.” (Heyting 1934, p. 14).

${ }^{8}$ In a later paper Heyting takes up the notion of inference step, and says, "The steps of the proof are the same as the steps of the mathematical construction" (Heyting 1958).
}

epistemic connotation of justifying an assertion: the proof of a proposition $A$ is the act of finding the construction intended by $A$, and to assert $A$ is just to affirm that the construction has been found; hence the proof justifies the assertion.

The term "construction" occurs frequently in Heyting's writing, and, as urged by Sundholm, one should distinguish between different senses of that word, among them "process of construction" and "object obtained as the result of a process of construction". ${ }^{9}$ Heyting uses the term in at least both these two senses, but I think that it is usually clear which one is intended. When he explains what a proof is it seems clear that he has in mind a construction in the sense of a process: to realize the intention expressed by a proposition $A$ is to perform a construction act, which may proceed in steps (cf. footnote 8), and which results in the construction of a mathematical object, namely the construction intended by $A$, thereby making the assertion of $A$ warranted.

However, in some places he uses the term proof of a proposition $A$ where it would had been more appropriate to speak of the construction intended by $A .{ }^{10}$ This ambiguous use of the word proof is also found in the well-known BHKexplanation of the logical constants formulated by Troelstra (1977) and Troelstra and van Dalen (1988), which "tells us the meaning of 'proof of a compound statement' in terms of 'proof of a constituent"" 11 . In that way we are given inductive clauses in a definition of what it is to be a "proof" of a sentence in the language of first order predicate logic, again without reference to inferences. However, the explanation, which is deliberately informal, is unclear about whether a "proof" of $A$ still proves $A$ or has now become instead the construction intended by $A$; some formulations (telling e.g. how a proof is given) seem to indicate the former, but other formulations seem to be better compatible with thinking of the term "proof" as standing rather for Heyting's constructions intended by propositions.

Heyting's notion of construction intended by a proposition was further developed by Howard. ${ }^{12} \mathrm{He}$ set up an enriched $\lambda$-calculus with terms typed by formulas in first order Heyting arithmetic (HA), in which a term of type $A$

\footnotetext{
9 Sundholm (1983, p. 164).

${ }^{10}$ For instance, he says, "an implication $A \rightarrow B$ means the intention of a construction that from any proof of $A$ leads to a proof of $B$ " (Heyting 1934, p. 14). Here it seems more appropriate to say: a construction that takes an arbitrary construction for (or intended by) $A$ to a construction for (or intended by) $B$. At least, this is how we now think of the construction for an implication-an operation that takes constructions to constructions. By this I do not want to deny that it can make sense to speak of a construction that leads from any process realizing the intention expressed by $A$ to a process realizing the intention expressed by $B$.

11 Troelstra (1977, p. 977).

12 In the paper "The formulae-as-types notion of construction" circulated privately from 1969 and appearing in print in Howard (1980).
} 
can be interpreted as denoting a construction intended by the proposition expressed by $A$. For each formula $A$ provable in HA there is such a term of type $A$ and vice versa; in fact, the terms are isomorphic to natural deductions, a result known as the Curry-Howard isomorphism.

This idea is developed much further in Martin-Löf's intuitionistic type theory, ${ }^{13}$ where the construction $a$ for ${ }^{14}$ a proposition $A$ and the process of forming such a construction are clearly distinguished. Even the process is exhibited, and its result, a construction $a$ for $A$ is recorded in a judgement of the form " $a: A$ ". The steps in the construction process, recorded by such judgements, are at the same time inference steps that together make up a proof of the judgment $a: A$.

As for terminology, the word "proof" is used in the type theory for constructions too; a judgement " $a$ : $A$ " being read " $a$ is a proof of the proposition $A$ ". In this way, the theory speaks of proofs of two kinds: proofs of propositions, which correspond to Heyting's constructions intended by propositions, and proofs of judgements. The latter shows at the same time how proofs of the first kind are constructed; proofs in Heyting's sense, construction processes, are thereby implicitly exhibited, as already noted. Proofs of the first kind, proofs of propositions, are not proofs in the epistemic sense, ${ }^{15}$ and to call them proofs is therefore misleading; they are better referred to as constructions or proof-objects. ${ }^{16}$ Proofs of the second kind, proofs of judgements, are genuine epistemic proofs, but they depend on the validity of the inferences they are built up of. So we must conclude that in neither case are we here provided with an epistemic notion of proof explained without reference to valid inferences.

It seems to remain however that Heyting has introduced a notion of proof without referring to valid inferences which enjoys the epistemic property that a proof of a proposition $A$ justifies the assertion of $A$. Proofs in Heyting's sense deviate from ordinary proofs in not being even concerned with inferences, being instead construction processes. It is thereby a different concept of proof than the one I would like to explain. Furthermore, if one makes assertions about the objects obtained in a construction process, they need to be proved too, and then it seems that one is led to proofs of judgements as in Martin-Löf's type theory. It seems

\footnotetext{
13 First stated by Martin-Löf (1975) and more fully presented by Martin-Löf (1984).

${ }^{14}$ Following a suggestion by Göran Sundholm, I say "construction for a proposition" instead of "construction of a propositions" since the latter expression would be ambiguous.

15 This was stressed by Martin-Löf (1998).

16 Martin-Löf (1984) uses the word construction when confusion with proof of judgements might occur. The label "proof-object" was suggested by Diller and Troelstra (1984), and is nowadays used frequently also by Martin-Löf.
}

therefore that, in the end, intuitionism cannot dispense with proofs in the usual sense.

For all that it may be claimed that as long as one is asserting ordinary propositions, understood intuitionistically but not explicitly referring to constructions, the assertions are justified just by constructing the intended constructions, that is, without proving in addition that the constructions found are of the required kind. This position, which as explained above seems to have been Heyting's, has been met with at least implicit objections. At an early stage, Kreisel (1962) took what he called a proof of an implication $A \rightarrow B$ to consist, not only "of a construction $c$ that transforms any proof of $A$ into a proof of $B$ ", but also of "a proof of the fact that $c$ has this property". Although Kreisel must be understood here as speaking of the construction for $A \rightarrow B$, a consequence of what he proposes is that to get a proof of an implication in Heyting's sense it is not enough to find a construction $c$ that transforms any construction for $A$ into a construction for $B$, one must also prove that the found construction has this property.

In Martin-Löf's type theory, there is no additional clause of Kreisel's kind in the condition for something to be a proof of (i.e. construction for) a proposition, but a judgement to the effect that a proposition $A$ is true can only be arrived at after a judgement of the form $a: A$ has been demonstrated.

Sundholm (1983) notes that Kreisel's idea had no effect on Heyting, who never makes the addition proposed by Kreisel (except when speaking of contributions by Kreisel) and insisted in a late statement, "I may assert $A \rightarrow B$ when I am able to convert any proof of $A$ into a proof of $B$ "17.

Is Heyting's view that the finding of the construction for a proposition justifies its assertion tenable? Before returning to that question I shall explore further some of the consequences of Heyting's view. His view does not lead to a general account of the concept of proof, but it offers an explanation of what it is to justify the assertion of a proposition without using the concept of proof, which was the problem raised in the previous section.

\section{Epistemic Grounds for Assertions}

Grounds are of interest both in metaphysics and in epistemology. Here we are concerned with grounds for assertions from an epistemic point of view. As already noted (Sect. 2), epistemic grounds can take many forms, but, in general, to have a ground for an assertion is to have made an act that amounts to being justified in making the assertion. We say for instance that we have a ground for an observational statement when we have made the relevant observation. In this

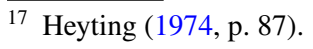


case the observation is gone after having been made, only the memory of it may remain. In the case of having a ground for the assertion of an arithmetical identity because of having made the relevant calculation, one may have recorded the steps of the calculation, and one has then a ground for the assertion also in the concrete sense of a protocol of the justifying act open for inspection. ${ }^{18}$

The process by which one finds a construction $a$ for a proposition $A$ can be recorded too. The resulting protocol can be seen as a description of not only the process but also of the obtained object $a$. We can say that one gets in possession of $a$ by describing it. Given Heyting's view that one gets justified in asserting $A$ by finding the construction $a$, we may also say that by this description one gets in possession of a ground for asserting $A$. Constructions for propositions may in other words be looked upon as grounds for the assertions of the propositions, although, of course, it is not the construction objects in themselves that make one justified in making assertions but the acts by which one gets in possession of them. Let us now look in more detail at these constructions for propositions, in terms of which one is explaining what it is to have grounds for assertions.

\subsection{Constructions for First Order Propositions}

I shall restrict myself here to considering propositions of a first order language, assuming a given individual domain $D$ and that it has been given what counts as constructions for atomic propositions. As described by Howard and MartinLöf, ${ }^{19}$ the constructions for compound propositions are generated by certain operations on constructions or on other operations that have $D$ or constructions as domains and constructions as ranges.

What is important for the understanding of a compound proposition $A$ is the condition that must be satisfied in order for there to be a construction for $A$; what exactly a construction for $A$ is taken to consist of is of less importance. For instance, it is commonly said that the construction for a conjunction $A \wedge B$ is an ordered pair whose elements are constructions for $A$ and for $B$, respectively, but the important point is that there is an operation that applied to constructions for $A$ and for $B$ yields a construction for $A \wedge B$. The operation may be called $\wedge \mathrm{I}$, and its value when applied to $\alpha$ and $\beta$ may then be written $\wedge \mathrm{I}(\alpha, \beta)$ and does not need to be defined further.

\footnotetext{
18 The protocol of a construction process is counted by Sundholm as a third sense of the word construction (cf. fn. 9).

19 See footnotes 12 and 13 for references. The account here will not follow exactly Howard's or Martin-Löf 's accounts and is also a little different from the one that I have presented earlier (Prawitz 2015a), but they all come essentially to the same in the respects that are relevant here.
}

We may therefore simply postulate that for each logical constant $\Phi$ except $\perp$ there is a primitive operation labelled $\Phi \mathrm{I}$ for forming constructions for compound propositions that have $\Phi$ as their main (outer) constant. For $\vee$ there will be two operations $\vee I_{1}$ and $V I_{2}$. Given constructions for atomic propositions and the operations $\Phi \mathrm{I}$, other operations on constructions can be defined. It is to be demanded of such a construction that its domain consists of all constructions for some proposition and that its value is specified constructively for each argument in the domain and falls within the range of constructions for some proposition. We shall also consider constructively specified operations on the individual domain $D$, and for such an operation it will be demanded that for some open proposition $A(x)$ its value when applied to an individual $a$ in $D$ is a construction for $A(a)$; its range varies in this way with the argument to which it is applied.

We can state the condition for something to be a construction for a compound first order proposition $A$ as follows:

$(\wedge \mathrm{I}) \gamma$ is a construction for the proposition $A \wedge B$, if and only, if $\gamma=\wedge \mathrm{I}(\alpha, \beta)$ for some constructions $\alpha$ and $\beta$ for $A$ and $B$, respectively.

(VI) $\gamma$ is a construction for the proposition $A_{1} \vee A_{2}$, if and only if, $\gamma=\mathrm{VI}_{\mathrm{i}}(\alpha)$ for some construction $\alpha$ for $A_{i}$ where $i=1$ or 2 .

$(\rightarrow \mathrm{I}) \gamma$ is a construction for the proposition $A \rightarrow B$, if and only if, $\gamma=\rightarrow \mathrm{I}(\alpha)$ for some operation $\alpha$ from constructions for $A$ to constructions for $B$.

$(\forall \mathrm{I}) \gamma$ is a construction for the proposition $\forall x A(x)$, if and only if, $\gamma=\forall \mathrm{I}(\alpha)$ for some operation $\alpha$ from individuals $a$ in the domain $D$ to constructions for $A(a)$.

( $\exists I) \gamma$ is a construction for the proposition $\exists x A(x)$, if and only if, $\gamma=\exists \mathrm{I}(a, \alpha)$ for some individual $a$ in the domain $D$ and some construction $\alpha$ for $A(a)$.

The constructions generated by the if-clauses are to be understood as given in this way uniquely. Thus, for instance, two constructions $\wedge \mathrm{I}\left(\alpha_{1}, \alpha_{2}\right)$ and $\wedge \mathrm{I}\left(\beta_{1}, \beta_{2}\right)$ for a conjunction are equal only if $\alpha_{1}=\beta_{1}$ and $\alpha_{2}=\beta_{2}$.

Since the condition for something to be a construction for a compound proposition $A$ is stated in terms of constructions of the constituents of $A$, what it is to be a construction for a first order proposition is defined inductively by the if-clauses above, given the constructions for atomic propositions.

\subsection{Grounds for Assertions Under Assumptions and for Open Assertions}

Given the constructions for atomic propositions and the primitive $\Phi I$-operations, we can define new operations from grounds to grounds that can be taken to be grounds for assertions under assumptions. For instance, we can define an operation $\wedge \mathrm{E}_{1}$ from grounds for asserting a proposition $A \wedge B$ to grounds for asserting $A$ specified by saying that when applied to a ground $\wedge \mathrm{I}(\alpha, \beta)$ it yields $\alpha$ as value. This 
operation, which can also be denoted by the expression $\wedge \mathrm{E}_{1}(\xi)$, where $\xi$ ranges over grounds for asserting $A \wedge B$, is taken to constitute a ground for asserting $A$ under the assumption $A \wedge B$.

To define such operations is an essential part in forming grounds for assertions since they are what the primitive operation $\rightarrow \mathrm{I}$ is applied to. For instance, a ground for asserting a proposition $(A \wedge B) \rightarrow A$ is formed by applying $\rightarrow \mathrm{I}$ to the operation $\wedge \mathrm{E}_{1}(\xi)$.

Operations defined on the individual domain $D$ that applied to an individual $a$ give a ground for asserting the proposition $A(a)$ play a similar role as arguments for the primitive operation $\forall \mathrm{I}$, and can be seen as grounds for asserting the open proposition $A(x)$.

\subsection{Constructions as Truth-Makers}

If we identify the truth of a proposition $A$ with the existence of a construction for $A$-an identification made e.g. by Martin-Löf $(1984,1994)$ - the existence of a construction $\gamma$ that satisfies the definiendum in the equivalence $\Phi I$ stated above can also bee seen as the truth condition of propositions that have $\Phi$ as their main constant. We can then see a construction for a proposition $A$ as a truth-maker of $A$ : a true proposition $A$ is true in virtue of the existence of a construction for $A .{ }^{20}$

The intuitionistic meaning theory for mathematical propositions first proposed by Heyting, according to which the meaning of a mathematical proposition $A$ is laid down by what counts as a construction for $A$ (or the construction intended by $A$ ), is often seen as something radically different from the truth conditional meaning theory that we are used to for classical logic since Frege. The difference is not, however, that classical logicians identify the meaning of proposition with its truth condition while intuitionists identify the meaning of a proposition by means of something quite different, which they call constructions; as we see, the two meaning theories can both be seen as truthconditional. ${ }^{21}$ Nor is it incompatible with classical logic to take the meaning of first order propositions to be determined by constructions; it is only that the condition for something to be a construction for a proposition has to be put differently for some forms of propositions, if not also the meta-language is classical. ${ }^{22}$

The truth conditions appearing in the intuitionistic meaning theory can be seen as a kind of constructivization of the usual classical truth conditions. The main difference between the two kinds of meaning theory is that classical

\footnotetext{
20 This was first proposed by Sundholm (1994).

${ }^{21}$ For an early emphasis of this point see Martin-Löf (1987).

${ }^{22}$ See Prawitz (2015b).
}

truth conditions, as usually stated, are in terms of states of things that may be inaccessible to us while intuitionistic truth conditions are stated in terms of constructions that we can in principle create and experience; this was a point emphasized by Heyting, who contrasted classical explanations of meaning that refer to imagined states of things thought of as existing independently of us with intuitionistic explanations that refer to constructions "thought of as a possible experience". ${ }^{23}$ Put shortly, intuitionistic truth-makers can in principle be constructed by us while classical truthmakers as they appear in realistic meaning theories may be inaccessible to us.

\subsection{Constructions as Grounds for Assertions}

This accessibility of constructions for propositions is what makes it possible to think of them as grounds for assertions in the sense described above (beginning of Sect. 4). Whether a speaker is justified in making an assertion must of course depend on conditions that refer to the speaker. Classical truth conditions, being formulated in terms of states of things that are independent of the speaker, cannot therefore be used to say what it is to be justified in asserting a proposition.

In contrast, intuitionistic truth conditions, being formulated in terms of objects that are in principle possible to construct and thereby to get in possession of, are easily reformulated as assertibility conditions, conditions for being justified in asserting propositions. In later publications, Heyting chose to use this alternative, thus explaining the meanings of the logical constants by stating such assertibility conditions for compound propositions of various forms. ${ }^{24}$

This gives a new perspective on the intuitionistic meaning theory. It can now be seen to be based on epistemic concepts, to be what Dummett called a justificationist meaning theory: the meaning of a proposition is determined by what it is to be justified in asserting the proposition, in other words, in terms of what it is to get in the possession of a ground for the assertion of the proposition.

When seeing constructions as grounds for assertions, an operation $\Phi I$ may be interpreted as an operation that we apply to given grounds and that then results in a ground for asserting a proposition whose main logical constant is $\Phi$. For instance, $\wedge \mathrm{I}$ can be understood as an operation that brings together grounds for asserting two propositions and

\footnotetext{
23 "als möglich gedachtes Erlebnis" (Heyting 1931, p. 113).

${ }^{24}$ See Heyting (1956). It is to be noted however that although, for instance, we can state the condition for asserting a conjunction or disjunction in terms of conditions for asserting the conjuncts or disjuncts, the condition for asserting an implication is not given in terms of conditions for asserting the antecedent and the consequent. In contrast, what it is to be a construction for a compound proposition is always given in terms of constructions for constituent propositions.
} 
yields as a result a ground for asserting their conjunction. That there is such an operation is taken to be constitutive for the meaning that we attach to conjunctions. Similarly, that there is an operation on grounds for asserting the proposition $B$ under the assumption $A$ that yields a ground for asserting $A \rightarrow B$ is taken to be constitutive for the meaning attached to an implication $A \rightarrow B$.

As seen, the operations for forming grounds for asserting compound propositions have the same structure as Gentzen's introduction rules (I-rules) in Natural Deduction; therefore the labels $\Phi I$. Gentzen's idea that the meanings of the logical constants are determined by their I-rules now comes to look very similar to Heyting's idea of how the meaning of a proposition is determined-in both cases, meaning is determined by what establishes assertions.

\subsection{Language with Terms Denoting Grounds}

To define operations on the domain $D$ or on grounds we need to describe what values they assume when applied to their arguments, and in general to be in possession of a ground for an assertion (in the form of a construction for the proposition) we must have a description of how the ground is constructed. This must be given in a language in which there are terms for denoting grounds.

A language suitable for that purpose should of course contain names for the primitive operations that have already been named $\Phi \mathrm{I}$, where $\Phi$ is one of logical constants; when speaking about the language used for describing grounds we may use such names autonomously. We need also names for the given grounds for asserting certain atomic propositions. To name operations on the given individual domain $D$ or on grounds for asserting various propositions, it is convenient to have open terms with free variables that range over individuals or grounds. I shall use $x, y, x_{1} \ldots$ as variables for individuals and $\xi, \zeta, \xi_{1}, \ldots$ as variables for grounds. The latter will be typed by propositions or open propositions. A variable $\xi^{A}$ ranges over grounds for asserting the proposition $A$, while a variable $\xi^{A(x)}$ can assume as value a ground for asserting $A(a)$ when $x$ assumes as value an individual $a$.

When applying $\rightarrow \mathrm{I}$ or $\forall \mathrm{I}$ to open terms with more than one free variable we need to distinguish the variables that are to remain standing as parameters and the variable that indicates the argument places of the operation on which we apply $\rightarrow \mathrm{I}$ or $\forall \mathrm{I}$, and I shall follow the usual convention to indicate this by writing the latter variable after the operation $\rightarrow \mathrm{I}$ or $\forall \mathrm{I}$, which is said to bind this variable. In this way we may also distinguish two quite different grounds for asserting a proposition such as $\mathrm{A} \rightarrow(\mathrm{A} \rightarrow \mathrm{A})$ by writing $\left(\rightarrow \mathrm{I} \xi^{\mathrm{A}}\right)\left(\rightarrow \mathrm{I} \zeta^{\mathrm{A}}\right) \zeta^{\mathrm{A}}$ and $\left(\rightarrow \mathrm{I} \xi^{\mathrm{A}}\right)\left(\rightarrow \mathrm{I} \zeta^{\mathrm{A}}\right) \xi^{\mathrm{A}}$, respectively.

Having typed the variables that range over grounds, each term can be typed in an obvious way so that a closed term of type $A$ denotes a ground for asserting the proposition $A$.
An open term of type $A$ with free variables $\xi_{1}, \xi_{2}, \ldots$, and $\xi_{n}$ typed by propositions $A_{1}, A_{2}, \ldots$, and $A_{n}$ denotes a ground for asserting the proposition $A$ under the assumptions $A_{1}, A_{2}, \ldots$, and $A_{n}$; if closed terms denoting grounds for $A_{1}, A_{2}, \ldots$, and $A_{n}$ are substituted for $\xi_{1}, \xi_{2}, \ldots$, and $\xi_{n}$, the resulting term denotes a ground for $A$. Similar explanations apply to terms with free individual variables.

When defining operations on individuals or grounds in the process of forming grounds, we need also to introduce names for these operations in order to have names for the grounds that are formed. The operation $\wedge E_{1}$ is a simple example that was given above. These operations must give rise to grounds for specific propositions so that terms in which names for the operations occur can also be typed.

To be in possession of a construction $\alpha$ for a proposition $A$, and thus of a ground for asserting $A$, is to have formed within a language of the kind indicated above a term $T$ that denotes $\alpha$, in other words, a term $T$ of type $A$. One has of course to know the meaning of the term, including the meanings of the names of the defined operations occurring in the term, that is, know how the operations in question are carried out, in order to be said to be in possession of the ground denoted by the term.

\section{Inferences as Operations on Grounds}

If one accepts the concept of ground considered in the previous section, agreeing that to get in possession of a ground for an assertion by constructing it is to become justified in making the assertion, there should be good prospects of defining an adequate concept of valid inference without getting into the circularity that we ended up in earlier.

Indeed, given this explanation of what it is for an inference to be justified, the definition of legitimate inference stated previously (Sect. 2) seems to catch without circularity what should be required of a valid inference. Formulated in terms of grounds instead, it would read: an inference is legitimate, if and only if, by performing the inference one gets in possession of a ground for the conclusion given that one had grounds for the premisses.

Admittedly, in view of the fact that natural deductions are understood as representing proofs and the isomorphism between the intuitionistic natural deductions and terms denoting grounds built up by the use of signs for the $\Phi \mathrm{I}$-operations and for defined operations corresponding to Gentzen's elimination rules, these terms can be interpreted as standing for proofs. But that is not their intended interpretation, which has now been explained without using the concept of proof.

However, we would like to understand how there can be legitimate inferences. So far we have only said that a reflective inference involves a transition from premisses 
to conclusion indicating that the conclusion is taken as being supported by the premisses. Clearly, an inference must involve something more than such a transition, a mere speech act, if it is really to yield a ground for the conclusion under the assumption that grounds are available for the premisses. It cannot be that a person justifies the conclusion of an inference by simply enunciating the conclusion after enunciating the premisses, indicating that she regards the conclusion to be supported by the premisses.

Phenomenologically we often experience a reflective inference as involving an operation by which we come to "see" that the proposition in the conclusion holds in view of the fact the propositions in the premisses do. What is of interest for us here is not, however, what is going on mentally when we infer but the idea that an inference involves an operation that results in evidence for the conclusion. The main point should be that the operation actually yields a ground for the conclusion when applied to grounds for the premisses.

My suggestion is that a (deductively) valid inference is understood as a transition from premisses to conclusion accompanied with the application of an operation to grounds that one takes oneself to have for the premisses such that it yields a ground for the conclusion whenever it is applied to grounds for the premisses. ${ }^{25}$

It then becomes literally true that when making a valid inference from premisses for which one has grounds one gets in possession of a ground for the conclusion. Furthermore, a proof consisting of a chain of valid inferences will provide a ground for its final conclusion if it starts from initial premisses to which grounds for them are attached. ${ }^{26}$

An inference figure (as defined in Sect. 2) together with an operation $\Theta$ on grounds can be defined as valid when $\Theta$ applied to any grounds for the assertion of $A_{1}, A_{2}, \ldots, A_{n}$ yields a ground for the assertion of $A$.

An application of Gentzen's introduction rule for the logical constant $\Phi$ then becomes a valid inference figure when it is joined with the $\Phi \mathrm{I}$-operation. To each inference figure obtained by applying one of his elimination rules we

\footnotetext{
25 Previously I have suggested that all inferences are to be understood as involving applications of operations on the grounds that one takes oneself to have for the premisses, and that a valid inference is an inference such that this operation always yields a ground for the conclusion when applied to grounds for the premisses (see e.g. Prawitz 2015a). But there is no need for such a reconstruction of inferences in general. Invalid inferences may occur in all kinds of ways and we may leave that open here. The problem is to account for reflective valid inferences in a non-circular way so that proofs consisting of them come to justify their final conclusions.

26 An initial premiss of a proof may be understood as obtained by an inference without premisses. That such a premiss is supplied with a ground for it comes then out as a special case of an inference being valid.
}

can associate an easily defined operation so that the combination becomes valid; for instance, an inference figure with $A \wedge B$ as premiss and $A$ as conclusion gets valid when assigned the operation $\wedge \mathrm{E}_{1}$ defined above, and an inference figure with the premisses $A \rightarrow B$ and $A$ and the conclusion $B$ becomes valid when assigned the operation $\rightarrow$ E defined by the equation

$\rightarrow \mathrm{E}\left(\left(\rightarrow \mathrm{I} \xi^{\mathrm{A}}\right) \mathrm{T}(\xi), \mathrm{U}\right)=\mathrm{T}(\mathrm{U} / \xi)$

where $T$ is to be of type $B$ and $U$ is to be of type $A$.

\section{Concluding Discussion}

As the involved notions have now been defined, when you perform a valid inference applied to premisses for which you have grounds, you get in fact a ground for the conclusion, regardless of whether you know or have proved that you have such a ground. To have a ground is in that sense an objective notion-you have it or do not have it independently of what you think about it. But as already stressed, it is the result of an activity: one gets in possession of a ground for an inferred assertion in the way suggested here by forming a term whose meaning one knows and whose denotation is in fact a ground for the assertion. The term is formed concurrently with the proof of the assertion in question. The inferences made in the proof deliver successively grounds for their conclusions, and the proof delivers in that way a ground for its final conclusion.

As noted above (Sect. 3), this idea has met more or less explicit objections according to which an assertion is not justified by just finding a construction of the proposition in question-it must also be proved that what has been found is in fact such a construction. On that view, one will of course reject the definition of ground for an assertion proposed above, and maintain instead that a real ground for an assertion of a proposition $A$ consists, not in a construction $a$ for $A$, but in a proof of a judgement which expresses for some construction $a$ that it is a construction for $A$. One is then back to a situation where the epistemic notion of ground is based on the notion of proof.

On behalf of the view that it suffices to find a construction for a proposition to be justified in asserting the proposition, it may be argued that one should distinguish between on the one hand asserting a proposition $A$ and on the other hand asserting that $A$ is true or that so and so is a construction for $A$. To assert that $A$ is true is from a constructive point of view to assert that there is a construction for $A$, which of course requires that one can specify a construction $a$ for $A$. To assert a proposition $A$, I take to be the same as uttering in assertive mood a sentence that expresses $A$. It certainly requires the speaker to have a construction for $A$ since, as always, she needs a ground for the assertion. But it does 
not require, one may argue, a proof that so and so is a construction for $A$, because that there is a construction of $A$ is not what she asserts; it is not a part of the content of the proposition $A$-for instance, the proposition that there are infinitely many primes is about primes, not about the existence of grounds.

Nevertheless, the inferences of a proof are what I have called reflective, which means that when making them one takes them to provide grounds for their conclusions. If such a belief is challenged, one must be able to meet the challenge somehow; it seems reasonable to say that otherwise the alleged proof must be withdrawn. ${ }^{27}$ When the inference is valid, as I have now defined it, there is an immediate first answer to the challenge: one can point to the result of the operation that is required to be present in a valid inference; more precisely one can exhibit a term $\Theta\left(T_{1}, T_{2}, \ldots, T_{n}\right)$ that denotes a ground for the conclusion provided that $T_{1}, T_{2}, \ldots, T_{n}$ denote grounds for the premisses from which the conclusion is inferred.

But, of course, the claim that the term denotes a ground for the conclusion can also be challenged. Although it would lead to a regress to require that an inference be proved to be valid before being used, it can be demanded that the belief that a used inference is valid can be motivated post factum. Now, there are two possible cases: either $\Theta$ is one of the primitive operations labelled $\Phi I$ where $\Phi$ is a logical constant or $\Theta$ is a defined operation. In the first case, the meaning of the proposition asserted by the conclusion is explained by saying that a construction for the proposition, and hence a ground for the conclusion, is generated by applying $\Phi \mathrm{I}$ to appropriate arguments. For instance, let $\Phi \mathrm{I}$ be $\wedge \mathrm{I}$. In this case the valid inference in question is a transition to the assertion of a conjunction $A \wedge B$ from the assertion of $A$ and the assertion of $B$, and the term claimed to denote a ground for the conclusion has the form $\wedge \mathrm{I}(T, U)$ where $T$ and $U$ are claimed to denote grounds for the two premisses. Knowing the meaning of $A \wedge B$, one knows that $\wedge \mathrm{I}(T, U)$ denotes a ground for the conclusion provided that $T$ and $U$ denote grounds for the two premisses.

In the second case, one has instead to refer to how the operation $\Theta$ has been defined. For instance, consider the case when $\Theta$ is $\wedge E_{1}$ and is accompanying a transition from the assertion of $A \wedge B$ to the assertion of $A$. From the definition of $\wedge \mathrm{E}_{1}$ and the fact that if $\gamma$ is a ground for the assertion of a conjunction there are unique grounds $\alpha$ and $\beta$ for the assertions of the two conjuncts such that $\gamma=\wedge \mathrm{I}(\alpha, \beta)$, it follows immediately that $\wedge E_{1}$ is an operation and that $\wedge E_{1}(\gamma)$ is a ground for the conclusion asserting $A$, when $\gamma$ is a ground for asserting $A \wedge B$, the premiss.

\footnotetext{
27 This is a theme that is elaborated in the paper by Cesare Cozzo in this volume.
}

But consider the case when $\Theta$ is instead defined as a unary operation from constructions for a proposition $A$ by saying that $\Theta(\alpha)$ is the construction denoted by the term exhibited by a certain Turing machine when it stops after having been started with a term that denotes $\alpha$, and assume that the output of the Turing machine is always a term denoting a construction for the proposition $B$ when the input is a term denoting a construction for $A$. Then, in fact, $\Theta$ satisfies the conditions imposed on operations, but since in contrast to the previous case it may not be obvious from its definition that it does so, nobody would regard $\Theta(T)$, where $T$ is known to be a term denoting a ground for asserting $A$, as a ground for asserting $B$ before it was proved that the Turing machine always behaves as assumed.

A restriction on the defined operations used in forming constructions for propositions is consequently required if the finding of a construction for a proposition is to be considered a ground for the assertion of the propositions. It was demanded above (Sect. 4.1) that the domain of an operation on constructions should consist of all constructions for a specific proposition $A$ and that the outcome of the operation should be a constructively specified construction for a given proposition $B$, but nothing was said about how it was to be known that this demand was fulfilled when the operation was used in the construction of a ground or as a part of a valid inference. If it had been required that it was proved that the demand was fulfilled, the notion of ground would again be depending on the concept of proof.

It is often said that the concept of proof should be defined in such a way that it becomes decidable whether something is a proof, but how this is to be achieved is seldom indicated, except of course in the case of formal proofs. The terms that denote constructions are supposed to be typed, and whether an expression has a type is decidable, but the rules for typing already assume that the demands put on the defined operations are fulfilled. What is needed in order to make it decidable whether something is a proof within the conceptual framework discussed here is a method to decide whether a proposed definition of an operation fulfills the demand that it is put on operations on constructions, and it is quite unclear how the latter question could be decidable.

The operation defined by a Turing machine considered above is certainly not accepted as an intuitionistic function unless there is a proof that the machine behaves as assumed in the example. What should be needed in order to get an explanation of the concepts of proof and valid inference along the lines suggested here is a general criterion for when a proposed definition of an operation on grounds guarantees that what is defined is really a total operation of the kind demanded here. 
Acknowledgements I am grateful to Cesare Cozzo, Per Martin-Löf, and Göran Sundholm for detailed and constructive comments to an earlier version of the paper.

Open Access This article is distributed under the terms of the Creative Commons Attribution 4.0 International License (http://creativecommons.org/licenses/by/4.0/), which permits unrestricted use, distribution, and reproduction in any medium, provided you give appropriate credit to the original author(s) and the source, provide a link to the Creative Commons license, and indicate if changes were made.

\section{References}

Diller J, Troelstra AS (1984) Realizability and intuitionistic logic. Synthese 60:253-282

Dummett M (1991) The logical basis of metaphysics. Duckworth, London

Heyting A (1931) Die intuitionistische Grundlegung der Mathematik. Erkenntnis 2:106-115

Heyting A (1934) Mathematische Grundlagenforschung, Intuitionismus, Beweistheorie. Springer, Berlin

Heyting A (1956) Intuitionism. An introduction. North-Holland Publisher, Amsterdam

Heyting A (1958) Intuitionism in mathematics. In: Klibansky R (ed) Philosophy in the mid-century. La Nuova Italia, Florence, pp 101-115

Heyting A (1974) Intuitionistic views on the nature of mathematics. Synthese 27:79-91

Howard W (1980) The formula-as-types notion of construction. In: Seldin J et al (eds) To H. B. Curry: essays on combinatory logic, lambda calculus and formalism. Academic Press, London, pp 479-490

Kreisel G (1962) Foundations of intuitionistic logic. In: Nagel E et al (eds) Logic, methodology and philosophy of science. Stanford University Press, Stanford, pp 198-212

Martin-Löf P (1975) An intuitionistic theory of types: predicative part. In: Rose HE, Shepherdson J (eds) Logic colloquium'73. North Holland, Amsterdam, pp 73-118

Martin-Löf P (1984) Intuitionistic type theory. Bibliopolis, Napoli

Martin-Löf P (1985) On the meanings of the logical constants and the justifications of the logical laws. In: Bernardi C, Pagli P (eds) Atti degli Incontri di Logica Matematica, vol. 2. Scuola di Specializzazione in Logica Matematica, Dipartimento di Matematica, Università di Siena, Siena, pp 203-281 (Republished in MartinLöf 1996)

Martin-Löf P (1987) Truth of a proposition, evidence of a judgement, validity of a proof. Synthese 73:407-420
Martin-Löf P (1994) Analytic and synthetic judgements in type theory. In: Paririni P (ed) Kant and contemporary epistemology. Kluwer, Dordrecht, pp 87-99

Martin-Löf P (1996) On the meanings of the logical constants and the justifications of the logical laws. Nordic J Philos Logic 1:11-60

Martin-Löf P (1998) Truth and knowability: on the principles C and K of Michael Dummett. In: Dales HG, Oliveri G (eds) Truth in Mathematics. Clarendon Press, Oxford, pp 105-114

Prawitz D (1973) Towards a foundation of general proof theory. In: Suppes $\mathrm{P}$ et al (eds) Logic, methodology and philosophy of science IV. North Holland, Amsterdam, pp 225-250

Prawitz D (2011) Proofs and perfect syllogisms. In: Cellucci C et al (eds) Logic and language. Cambridge Scholars, Newcastle upon Tyne, pp 385-402

Prawitz D (2015a) Explaining deductive inference. In: Wansing H (ed) Dag Prawitz on proofs and meaning. Outstanding contributions to logic, vol 7. Springer, Cham, pp 65-100

Prawitz D (2015b) Classical versus intuitionistic logic. In: Haeusler EH et al (eds) Why is this a proof? Festschrift for Luiz Carlos Pereira (Tributes volume 27). College Publications, Milton Keynes, pp 15-32

Prawitz D (2016) On the relation between Heyting's and Gentzen's approaches to meaning. In: Piecha T, Schroeder-Heister P (eds) Advances in proof-theoretic semantics. Springer, Cham, pp 5-25

Prawitz D (2018) The fundamental problem of proof theory. Studia Logica (forthcoming)

Ross WD (1949) Aristotle's prior and posterior analytics. Oxford University Press, Oxford

Schroeder-Heister P (2006) Validity concepts in proof-theoretic semantics. Synthese 148:525-571

Sundholm G (1983) Constructions, proofs and the meaning of logical constants. J Philos Log 12:151-172

Sundholm G (1994) Existence, proof and truth-making: a perspective on the intuitionistic conception of truth. Topoi 13(2):117-126

Sundholm G (1997) Implicit epistemic aspects of constructive logic. J Log Lang Inf 6:191-212

Troelstra AS (1977) Aspects of Constructive Mathematics. In: Barwise $\mathrm{J}$ (ed) Handbook of mathematical logic. North-Holland, Amsterdam, pp 1052-1973

Troelstra AS, van Dalen D (1988) Constructivism in mathematics, 1. North-Holland, Amsterdam 